\title{
A Simple Model for Growth of a Planar Hydrogenated Carbon Cluster under Interstellar or Circumstellar Conditions
}

\section{G. Pascoli}

Faculté des Sciences, Amiens, France

The physico-chemical origin of the hydrogenated carbon clusters (cumulenes, PAHs, graphite or amorphous carbon) in space is still an open question. We have worked out a numerical simulation code in order to build up planar (graphite-like) carbon clusters. We assume that hydrogen atoms can fix on the carbon skeleton following a random process allowing for $\mathrm{H}_{2}$ formation. The structures we have found are very complex. In a given cluster, several molecular entities can simultaneously be present: $\left(\mathrm{sp}_{2}\right)$ carbon chains, rings or compact formations (aromatic structures or small PAHs). We argue that these very contorted hydrogenated structures could be ubiquitous in the interstellar medium, in carbonrich circumstellar regions and in PNe. 